\title{
Pensar la integración regional desde los márgenes: la importancia y desafíos de la dimensión subestatal en América Latina desde la experiencia chileno-argentina*
}

\section{Considering regional integration from the margins: The importance and challenges of the sub-state dimension in Latin America based on the Chilean- Argentine experience}

\author{
Felipe Venegas San Martín ${ }^{* *}$ \\ Facultad de Ciencias Sociales, Universidad de Playa Ancha, Chile.
}

\begin{abstract}
Cómo citar este artículo: Venegas, F. (2019). Pensar la integración regional desde los márgenes: la importancia y desafíos de la dimensión subestatal en América Latina desde la experiencia chileno-argentina. Si Somos Americanos. Revista de Estudios Transfronterizos, 19(2), 69-88. DOI: http://dx.doi.org/10.4067/S0719-09482019000200069
\end{abstract}

\section{Resumen}

La integración ha sido estudiada y practicada desde la centralidad del Estado hacia mecanismos de integración supranacional, no obstante, nuestra realidad regional, determinada por la incapacidad de los Estados para desarrollar una integración profunda, ayuda a explicar el fracaso del proceso. Hoy, los estudios de integración y de frontera plantean invertir la relación vertical desde el Estado hacia lo local a través de la articulación de mecanismos subestatales, como los Comités de Frontera e Integración, los que por sus características deben ser considerados como el primer y clave eslabón de la cadena de

* El presente artículo forma parte de la investigación doctoral "Los desafíos de la integración regional. El lugar de la migración en Mercosur" presentada al Instituto de Estudios Avanzados de la Universidad de Santiago de Chile y cuenta con el apoyo de la beca de doctorado nacional CONICYT.

** Periodista, Universidad de Playa Ancha. Máster en Desarrollo Económico en América Latina, Universidad Internacional de Andalucía. Doctorando en Estudios Americanos, Universidad de Santiago de Chile. Facultad de Ciencias Sociales, Universidad de Playa Ancha, Valparaíso, Chile. Correo electrónico: fvenegas@upla.cl 
integración regional. Como hipótesis, en el artículo se plantea que los mecanismos de pequeña escala, como los Comités de Frontera e Integración, han demostrado mayor eficacia resolutiva y propositiva de problemáticas de frontera y más flexibilidad para adaptarse a los cambiantes escenarios de la geografía política regional. Sin embargo, no han alcanzado el suficiente nivel de autonomía respecto de las decisiones estatales centrales, y a su vez, más relevancia en la influencia y relación con el proceso regional, lo que pone en discusión la importancia de un enfoque que sea capaz de incorporar a la sociedad civil en las acciones paradiplomáticas.

Palabras clave: integración regional, fronteras, Chile-Argentina.

\begin{abstract}
Integration has been studied and practiced from the centrality of the State towards supranational integration mechanisms. However, our regional reality, which is shaped by the inability of States to develop strong integration, helps explain the failure of the process. Today, integration and border studies seek to reverse the vertical relationship from the State to the local through the articulation of sub-state mechanisms such as Border and Integration Committees, which based on their characteristics, should be considered the first and most important link in the regional integration chain. We propose that small-scale mechanisms such as Border and Integration Committees have proven effective for resolving and identifying border issues and are more flexible when it comes to adapting to the changing scenarios of the regional political landscape. However, they have not reached a sufficient level of autonomy with respect to key state decisions, and as such, have not become more relevant in regard to influencing and forging a relationship with the regional process. This highlights the importance of an approach that can include civil society in paradiplomatic actions.
\end{abstract}

Keywords: Regional integration, borders, Chile, Argentina.

\title{
Introducción
}

La integración regional en América Latina ha sido un proyecto permanente en la política internacional. Privilegiando el enfoque realista en las relaciones internacionales, los Estados han desarrollado iniciativas de integración mediante acuerdos regionales o subregionales (ALADI para el primer caso, MERCOSUR para el segundo). 
No obstante, hoy, dada la complejidad y multidimensionalidad de los problemas, debemos considerar una tercera dimensión: la integración a pequeña escala a través de espacios y regiones fronterizas mucho más vinculadas con la participación de las comunidades que las habitan y que no se relacionan necesariamente con los grandes acuerdos.

Las tres dimensiones no son excluyentes, sino complementarias y deben articularse para profundizar las interrelaciones y el intercambio, así como asumir aspectos que muchas veces no son abordados por los grandes acuerdos y que juegan un rol fundamental en la integración de los países sudamericanos. Así es posible la correspondencia entre las dimensiones macro y micro para el desarrollo de comunidades, provincias, regiones o departamentos alejados de los centros de toma de decisión, los que muchas veces dependen de manera importante de su comunidad transfronteriza para avanzar en el desarrollo.

En tal sentido han ido cobrando relevancia, especialmente en el Cono Sur, la creciente correlación, cuando no interdependencia de las comunidades de frontera, las que han institucionalizado sus relaciones de integración a través de los Comités de Frontera e Integración.

Se trata de espacios de encuentro, cooperación y solución de problemas en regiones fronterizas, que se caracterizan por su carácter local (crecientemente descentralizado) y por la participación ascendente de actores no estatales, condición que supera las iniciativas de paradiplomacia desarrolladas por órganos subestatales. Esto les confiere a estas instancias mayor flexibilidad y eficacia para abordar las relaciones transfronterizas y para incidir en los procesos de nivel supranacional, constituyéndose en una representación genuina de las comunidades en el proceso de integración regional.

En el caso de las relaciones chileno-argentinas, los comités de frontera y actualmente los de integración y desarrollo fronterizo han demostrado una vigorosa capacidad de integración y cooperación a ambos lados de la cordillera, ampliando sus ámbitos de acción e institucionalización y demostrando estabilidad a pesar de los vaivenes en las relaciones entre ambos países.

\section{La integración regional}

La integración regional ha sido a lo largo de la historia de América Latina un propósito fundamental, dadas las características de nuestra región como su historia; y también sus problemas sociales. Se puede entender esta como un proceso complejo de interacciones que persigue una finalidad positiva para los países y la comunidad internacional:

(A) Se trata de un desplazamiento hacia una mayor cooperación entre los estados;

(B) una transferencia gradual de autoridad de las instituciones supranacionales; 
(C) una homogeneización gradual de los valores; y

(D) la generación de una sociedad civil global y, con ella, la construcción de nuevas formas de comunidad política. (Griffiths, O'Callahan y Roach, 2002, p. 158)

De lo planteado por los autores, la integración es un proceso en el que las relaciones entre los Estados se van profundizando desde la homogeneización de valores hasta llegar a construir una nueva forma de comunidad política. Aunque pareciera en el fondo corresponder a nuevas formas de articulación política en el sentido del regionalismo planteado por Juan Carlos Puig (1986), un autor fundamental en el ámbito de la integración como Bela Balassa (1980) esboza una visión más amplia:

Algunos autores incluyen la integración social en el concepto, otros entienden diferentes formas de la cooperación internacional bajo este principio, y también se ha presentado el argumento de que la mera existencia de relaciones comerciales entre economías nacionales independientes es un signo de integración. (p. 1)

Por su parte, Karl Deutsch plantea que

la integración es una relación entre unidades en la cual éstas son mutuamente interdependientes y poseen en conjunto propiedades sistemáticas de las que carecerían si estuviesen aisladas. Un sistema integrado se encuentra cohesionado en la medida en que puede afrontar tensiones y presiones, soportar desequilibrios y resistir divisiones. Así entonces la integración es un proceso amplio, intenso y profundo que implica la vinculación e interpretación social, política, económica, cultural, científica, diplomática e incluso militar entre dos o más naciones. (1978, p. 285)

Por otro lado, el autor argentino Juan Carlos Puig (1986), uno de los principales pensadores de la teoría de la autonomía, define la integración como un fenómeno social según el cual dos o más grupos humanos adoptan una regulación común permanente de determinadas materias que hasta ese momento pertenecían a su exclusiva competencia. De esta definición se desprende su visión de la integración.

En primer lugar, al concebir la integración como un fenómeno social, Puig se aleja de aquellas visiones integracionistas centradas en el rol de los Estados nacionales para adoptar una visión más amplia que incluya también a "cualquier otra agrupación, micro (como sociedades y empresas) y macro (como la comunidad internacional)" (1986, p. 41). En este sentido, Puig no limita la acción de la integración a los Estados y a las élites gobernantes, sino que en su esquema de pensamiento aquella también debe ser expresión del sentir y querer de las poblaciones.

En segundo lugar, el propio autor resalta la importancia de la voluntad política al expresar que la integración "se trata de conductas que tienen como propósito que los grupos sociales en cuestión renuncien en determinadas materias a la actuación individual para hacerlo en forma conjunta y con sentido de permanencia" (Puig, 1986, p. 49). 
A lo largo de este trayecto latinoamericano de más de dos siglos, la integración se ha intentado desde diferentes enfoques, con sus respectivos mecanismos institucionales para llevarla a cabo, sin embargo, el propósito de una integración profunda, aunque sea bajo el propósito de acrecentar la liberalización y el comercio de nuestra región-como lo plantea Lawrence (1996), sigue siendo, en la actualidad, una tarea pendiente.

En términos tradicionales podemos identificar dos dimensiones o niveles para abordar la integración en América Latina: una, a través de los Estados en mecanismos regionales. Dos, mediante mecanismos subregionales de integración. Ejemplo del primer caso es la Asociación Latinoamericana de Integración (ALADI) y ejemplo del segundo, el Mercado Común del Sur (MERCOSUR).

\section{La dimensión estatocéntrica de la integración}

Tradicionalmente y de acuerdo con las teorías más clásicas de las relaciones internacionales, en especial las del realismo de Morgenthau (1986) y de Waltz (2008), únicamente el Estado actuaba y representaba al país en asuntos exteriores. Las negociaciones se llevaban a cabo a través de los ministerios de relaciones exteriores, se diagramaban los tratados y se coordinaban las reuniones entre los presidentes.

Si bien los mandatarios se involucraban de forma cada vez más directa, las visiones clásicas mantenían a la política exterior como una voz única, expresión de un ministerio de relaciones exteriores sobre el que recaía la responsabilidad casi exclusiva de mantener funcionando los engranajes de las vinculaciones internacionales de un país.

Esta dinámica estatocéntrica de las relaciones internacionales no es suficiente para enfrentar los desafíos de una sociedad internacional cada vez más compleja, que presenta una diversidad creciente de actores que demandan participación. Ya a partir de la década de 1970, con la teoría de la interdependencia compleja de Nye y Keohane (1988), se critica la mirada estatocéntrica por considerarla insuficiente y se propone la necesidad de incluir a nuevos actores que pudieran interactuar en diferentes niveles. En este sentido se comenzó a considerar en las relaciones internacionales a los actores privados del ámbito económico.

En el caso de América Latina, donde las relaciones internacionales le han otorgado un importante espacio al propósito integracionista, el paradigma estatocéntrico -en cuanto promotor y configurador de la integración- ha demostrado ser particularmente insuficiente, toda vez que el panorama político latinoamericano ha presentado una permanente debilidad institucional al interior de los Estados y en los propios mecanismos de integración.

Los abruptos cambios políticos, la desconfianza entre los países (alentada durante décadas por la Doctrina de Seguridad Nacional, a pesar de los esfuerzos posdictatoriales orientados a recuperar la confianza y cooperación, como el caso del MERCOSUR) y la fragilidad del Estado en el contexto neoliberal (Strange, 1996) -que llevó a plantear la integración desde 
un sesgo marcadamente economicista, sumado a la integración a los mercados mundiales por parte de las economías latinoamericanas-,hacen necesario no solo replantear el rol del Estado, sino fundamentalmente replantear el papel de la sociedad civil en las relaciones internacionales y particularmente en la integración, a través de mecanismos que superen la paradiplomacia, entendida tradicionalmente como la acción en el ámbito internacional de gobiernos no centrales, (Duchacek, 1990; Meirelles Ribeiro, 2008) o desde una perspectiva más relacionada con la idea de este trabajo, como propone Cornago (2014):

Entender a la paradiplomacia como una visión descentralizada de la diplomacia, menos encapsulada en la visión unidimensional del Estado como realidad espacial cerrada, y más atenta a su dimensión de relación social compleja, ofrece perspectivas mucho más prometedoras para gestionar los desafíos presentes y futuros de la agenda bilateral en la región. (2014, p. 14)

De esta forma, se buscaría fomentar la integración también a través de actores no estatales, especialmente comunitarios, surgidos desde la sociedad civil, en pos de superar las trabas político-burocráticas a las que se ven sometidos los actores paradiplomáticos. Es decir, entender la diplomacia contemporánea en su multidimensionalidad, progresivamente más alejada de la capacidad de gestión del Estado y más requeridora de la participación de agentes no estatales. Amilhat Szary (2016) señala categóricamente al respecto:

El alcance de la paradiplomacia concierne a un número cada día más creciente de personas, junto con incorporar una cantidad igualmente relevante de actores de gran diversidad; por ello su relevancia ampliada impone un examen teórico (...), de forma de llegar a proponer una redefinición crítica del fenómeno paradiplomático. (2016, pp. 47-48)

Es decir, la posibilidad de que el Estado defina los objetivos en política diplomática y que otros actores, ajenos al Ministerio de Relaciones Exteriores, puedan participar en las negociaciones, tal como ya se demuestra en el ámbito de los negocios.

Desde el punto de vista del interés académico por la política subnacional, desde la década de 1980, a partir de la publicación del número de otoño de 1984 de la revista Publius, en el artículo titulado "The international dimensions of subnational self government" de Ivo Duchacek, se señalaba la necesidad por parte de los estados federales de tener expresión externa en sus relaciones, que la idea de la inclusión de los gobiernos no centrales comenzó a prestar atención por parte de la comunidad académica, generando así un debate mundial respecto de lo que se conocía como dimensión subestatal de las relaciones internacionales, discusión de la que no estuvo exenta América Latina y que hoy requiere, como lo hemos expuesto, su ampliación a otros actores, dado el aumento y la diversidad de las relaciones transfronterizas. Pero primero nos proponemos profundizar en lo que se entiende tradicionalmente por dimensión subestatal, o paradiplomacia, y las diferentes perspectivas en torno a esta. 


\section{La dimensión subestatal de la integración, una discusión abierta}

Por dimensión subestatal de la integración nos referimos a todos los actores e instancias delegadas y/o descentralizadas del Estado que desarrollan relaciones internacionales de integración en diferentes niveles, como pueden ser los gobiernos locales, provinciales, regionales, los municipios, departamentos y comités binacionales surgidos a partir de la voluntad de los Estados, etc.

En ese sentido, según Rosenau (1997), en el sistema internacional actual conviven fuerzas fragmentadoras descentralizantes y fuerzas integradoras centralizantes. Este fenómeno al que denomina "fragmentación" (Rosenau, 1997, p. 70), impulsa una relocalización de la autoridad en dos direcciones: "hacia arriba", lo cual implica instancias supranacionales como organizaciones internacionales, entidades intergubernamentales regionales, entre otras, y/o "hacia abajo", en dirección de grupos subnacionales, dentro de los cuales se encuentran las minorías étnicas, religiosas, los gobiernos locales y las organizaciones con finalidades específicas.

Con la ampliación del interés hacia la función que podían cumplir actores no centrales del Estado se fue aceptando la idea de que actores subestatales (gobernaciones, municipios) pueden cumplir un rol no contrario ni paralelo al Estado, sino complementario, que se mueve en el núcleo blando de las relaciones internacionales y que produce un aporte a la integración, al ser instancias más flexibles que se acomodan mejor a los cambios en la geografía política regional, caracterizada por el excesivo centralismo de la mayoría de los países latinoamericanos, zonas fronterizas alejadas de los centros de decisión y una política exterior variable, estrechamente vinculada a la ideología de los gobiernos de turno.

Nos referimos a la complementariedad con el Estado, ya que aún es habitual encontrar en el debate las reservas que a este respecto han tenido expertos como Aldecoa y Keating (1999), al plantear lo que denominan "protodiplomacia", es decir, intentos de realizar una actividad internacional por parte de un actor subnacional que busca ser el germen de un proceso de secesión (del tipo del que se propone en Québec el Parti Québécois o los ejemplos de los gobiernos autónomos de Cataluña o del País Vasco en España).

Otra postura es la de Hocking (1993), para quien el término paradiplomacia presume implícitamente intereses incompatibles, lo que puede generar rechazo en actores que participan u observan (políticos, académicos, técnicos, ciudadanos): "As a consequence, rather than attempting to locate NCGs (No Central Governments) within the foreign policy processes alongside their national governments, there has been a strong presumption that each have incompatible interests and stand in opposition to one another" $(1993, \mathrm{p} .3)$. De ahí que este autor proponga el término diplomacia por múltiples capas/niveles, con el que intenta describir las vinculaciones y complementariedades entre la diplomacia estatal y no estatal. Para Hocking "[t]he nature of contemporary public policy with its dual domestic international features, creates a mutual dependency between the levels of government and 
an interest in devising cooperative mechanisms and strategies to promote the interests of each level" (1993, p. 4).

En este trabajo aceptamos que la paradiplomacia juega un rol en cuanto articuladora institucional de las iniciativas transfronterizas, lo cual no significa que esta instancia se establezca como la única posible, al contrario, debe impulsar las iniciativas no estatales de frontera, constituyéndose como un puente entre la sociedad civil y el Estado. Así lo indica Maira (2010) al referirse a la complementariedad entre lo central y lo local:

Se entiende que en la construcción de nuevos consensos pro regionales deberán participar fuerzas que actúen en un sentido vertical descendente como vertical ascendente propiciando así tanto la integración desde arriba (top-down) como la integración desde abajo (bottom up), es decir, la integración desde el ciudadano y de las autoridades políticas más cercanas al ciudadano. (2010, p. 17)

Por otra parte, de acuerdo con este mismo autor, los temores a la sustitución de la diplomacia estatal por la subestatal son menos reales en América Latina:

En los diversos países latinoamericanos, esta tendencia tiene diversos grados de desarrollo, pero siempre en base al reconocimiento de la unidad nacional y a un papel conductor de las relaciones internacionales por parte de los jefes de Estado y las cancillerías de cada país. (2010, p. 18)

Por lo tanto, las instancias de cooperación en esta escala subestatal representan una interesante posibilidad de articulación entre el Estado central y las zonas fronterizas más apartadas, lo que permite poner en la agenda temas no siempre detectados desde el nivel central y que pueden ser claves en la integración entre dos o más países, además de resistir mejor a los cambios de orientación en política internacional que se puedan producir en un país.

Esto se podría generar más fácilmente en los Estados de organización federal, donde estas unidades deberían contar con mayores prerrogativas que en los países de organización unitaria. No obstante, salvo Brasil, y de manera muy acotada, los Estados que constitucionalmente son federales, por ejemplo Argentina, Venezuela, en la práctica son bastante unitarios, lo que implica una dificultad de hecho en sus relaciones transfronterizas.

En el caso argentino, la creación de la Comisión Nacional de Fronteras en 2017, como un "[o]rganismo regulador y coordinador del funcionamiento de los Pasos Internacionales y Centros de Frontera que vinculan a la República Argentina con los países limítrofes, a fin de armonizar su funcionamiento desde una perspectiva interministerial que aumente la integración fronteriza" (Decreto 68/2017, Boletín Oficial de la República Argentina, 2017), centraliza en una instancia nacional y ministerial la gestión de las relaciones de frontera, omitiendo en su estructura a las organizaciones representativas de la sociedad civil. Su conformación (Art. $2^{\circ}$ ) deja bastante claro este punto, por cuanto establece que este Comité será conformado por: 
a) Secretario de Fronteras del Ministerio de Seguridad.

b) Secretario de Relaciones Exteriores del Ministerio de Relaciones Exteriores y Culto.

c) Secretario de Obras Públicas del Ministerio del Interior, Obras Públicas y Vivienda.

d) Secretario de Interior del Ministerio del Interior, Obras Públicas y Vivienda.

e) Secretario de Gestión de Transporte del Ministerio de Transporte.

f) Director General de la Dirección General de Aduanas dependiente de la Administración Federal de Ingresos Públicos, organismo descentralizado actuante en la órbita del Ministerio de Hacienda.

g) Director Nacional de la Dirección Nacional de Migraciones, organismo descentralizado actuante en la órbita del Ministerio del Interior, Obras Públicas y Vivienda.

h) Presidente del Servicio Nacional de Sanidad y Calidad Agroalimentaria, organismo descentralizado actuante en la órbita del Ministerio de Agroindustria.

i) Director Nacional de la Gendarmería Nacional Argentina dependiente del Ministerio de Seguridad.

j) Prefecto Nacional Naval de la Prefectura Naval Argentina dependiente del Ministerio de Seguridad.

k) Jefe de la Policía Federal Argentina dependiente del Ministerio de Seguridad.

I) Director Nacional de la Dirección Nacional de Registro, Fiscalización y Sanidad de Fronteras dependiente del Ministerio de Salud (Decreto 68/2017).

Se constata entonces que, a pesar del avance que significa para las relaciones internacionales en América Latina la irrupción de actores subestatales, es necesario señalar que se sigue tratando de instancias que representan (aunque descentralizadamente) al Estado y sus intereses tienden a mezclarse con los centrales, por lo que muchas veces no poseen atribuciones en su actuación internacional y/o se encuentran sometidos a distintos niveles de burocracia más o menos centralizada, lo que representa un desafío si el objetivo es integrar a los países latinoamericanos desde lo subestatal.

En el caso de Chile, la política subestatal en el ámbito internacional fue creada mediante la Orden de Servicio $N^{\circ} 145$, del 7 de junio de 2000, por la Dirección de Coordinación Regional (DICORE), instancia precaria en competencias y recursos, que la mantuvo como una instancia esencialmente débil. Tal como plantea Fuentes (2015), “dicha orgánica quedó a cargo de profesionales ajenos al servicio exterior, con una dotación mínima de personal y un presupuesto que incluía sólo los recursos básicos para su funcionamiento" (2015, p. 281). 
Sin embargo, de acuerdo con el mismo autor, el problema en el caso chileno parece ser más de fondo:

Tales circunstancias (la precariedad) pueden explicarse, entre otras razones, por el desconocimiento y falta de interés relativo sobre estas materias producto del atraso teórico, conceptual y práctico de nuestro Estado, así como la ausencia de una voluntad política mayor para impulsar cambios sustantivos. (Fuentes, 2015, p. 281)

En este punto es pertinente preguntarse qué tan representativos de las comunidades aisladas en zonas de frontera pueden ser los actores subestatales y qué capacidad tienen para articular propuestas y soluciones locales. Se hace necesario, entonces, potenciar la participación de los actores no estatales, como la dimensión de base que sustente desde abajo el proceso de integración regional.

Como señalan Tapia y González:

Lo que se está produciendo es una multiplicación de actores no estatales y de procesos transfronterizos que generan caminos en el alcance, la exclusividad y la competencia de la autoridad estatal sobre el territorio nacional. Esos nuevos actores y su impacto en los espacios fronterizos deben ser considerados para evitar interpretaciones en clave nacional que no se ajustan a la realidad. (2014, p. 19)

En definitiva, las instancias subestatales representan un avance, aunque aún precario en relación con las posibilidades de ampliar y potenciar las relaciones internacionales a nivel local. A nuestro entender, tres serían las causas principales que obstaculizan este propósito: (i) dependencia (más o menos estrecha) del Estado central; (ii) falta de atribuciones y competencias legales-institucionales; (iii) desconocimiento y desconfianza por parte de los actores políticos y sociales de la más amplia naturaleza, respecto de la importancia de la acción internacional de las unidades políticas locales.

\section{Los Comités de Frontera e Integración}

En las últimas décadas se ha avanzado en la articulación de relaciones entre los gobiernos locales de diferentes países a través de acciones de hermanamiento, cooperación y el trabajo de las Comisiones Binacionales Fronterizas. Sin embargo, la experiencia subestatal que ha tenido un desarrollo más dinámico en las relaciones internacionales de los países del Cono Sur, y especialmente entre Argentina y Chile, son los Comités de Frontera e Integración. ${ }^{1}$

1 No consideramos en este trabajo la experiencia de MERCOCIUDADES, al tratarse de una red de gobiernos locales de MERCOSUR que si bien es una iniciativa interesante de articulación local-regional, no es expresión de las ciudadanías locales, como lo son los Comités de Frontera y/o Integración, sino más bien de la relación Estados- Mercosur.

78 Si Somos Americanos. Revista de Estudios Transfronterizos 
Los Comités de Frontera e Integración conforman espacios de encuentro y trabajo entre representantes, delegados de los gobiernos locales en áreas y regiones fronterizas, presididos por los respectivos cónsules, que en una primera instancia abarcan diversos temas relacionados con la coyuntura local, tales como transportes, comercio, turismo, aduanas, entre otros. Progresivamente se han ido sumando representantes de ámbitos privados y de la sociedad civil, lo que les otorga un importante matiz temático respecto de las instancias subestatales.

Los comités constituyen mecanismos internacionales cuya función principal es resolver o bien encauzar problemáticas para su decisión a otros niveles, ya sean estos nacionales, regionales, provinciales, departamentales, etc. A este respecto, a pesar de la heterogeneidad en su funcionamiento, podemos distinguir dos ámbitos de atribución general: por una parte, en aquellos asuntos típicamente fronterizos cuya resolución se obtiene mediante la coordinación de decisiones de los propios funcionarios destinados en frontera; y un segundo nivel, de competencia nacional, respecto del cual los comités actúan transmitiendo la cuestión a los ministerios de relaciones exteriores, asesorando sobre su forma de resolución e interesándose en su seguimiento posterior.

Algunos de los temas tratados en los Comités de Fronteras e Integración han sido la facilitación fronteriza, desarrollo y coordinación de planes de infraestructura; la promoción de proyectos de turismo; el fomento de actividades compartidas de promoción de exportaciones e inversiones en el ámbito de las respectivas provincias y regiones; la vinculación y cooperación académica; la cooperación y complementación en las políticas de protección social, salud y equidad de género, entre otros. La experiencia indica que son los temas del primer ámbito los más tratados y, al mismo tiempo, por su naturaleza, los que obtienen una implementación más rápida, al no depender de instancias burocráticas, sobre todo en aquellos temas que requieren el involucramiento del nivel central del Estado.

La primera experiencia de comité de frontera corresponde a la zona de frontera entre Ciudad del Este (Paraguay) y Foz de Iguazú (Brasil). El comité fue creado en octubre de 1979 como uno de los acuerdos contenidos en el acta de la Decimosegunda reunión Paraguayo-Brasileña de los Organismos de Aplicación del Convenio de Transporte Terrestre de 1966 y cuya finalidad inicial estaba referida a la agilización del tráfico internacional por el puente Internacional de la Amistad.

Durante las décadas de 1980 y 1990, con el propósito de los gobiernos de revitalizar el proceso de integración se desarrollaron en el Cono Sur los siguientes Comités Fronterizos: Brasil-Paraguay (1987), Argentina-Paraguay (1985), Argentina-Chile (1987), Argentina-Uruguay (1986), Argentina-Bolivia (1987) y Argentina-Brasil 
(1990). ${ }^{2}$ Dada la eficacia de este instrumento para abordar determinados temas fronterizos, se ha producido una expansión progresiva de estas iniciativas, las que se han ido convirtiendo en espacios de desarrollo e integración que superan los límites de acción del Estado central. De acuerdo con Valenciano:

Un cambio de la función de la frontera que pasa de lo restrictivo de las líneas defensivas y propias de economías cerradas y sociedades escasamente vinculadas, a una frontera articulante en el marco de una integración bilateral o multilateral y de una creciente solidaridad internacional. Este nuevo marco supone una mayor movilidad de factores y participación de los sectores de la economía y de la sociedad fronteriza desvinculados de la interferencia que las antiguas fronteras políticas originaban en los territorios limítrofes. Da lugar a formas de vida sui géneris con una escala de valores particulares y en oportunidades con expectativas e intereses contradictorios respecto a los de sus países expresados globalmente o respecto de su centro administrativo. (1991, p. 10)

Aunque siguen constituyendo instancias paradiplomáticas, los Comités de Frontera han ido ampliando su radio de acción y, tal vez lo más importante, involucrando a organizaciones sociales de los más diversos ámbitos que en la mayor parte de los casos llevan sus agendas con amplia independencia del Estado, disminuyendo de esta forma la burocracia y aumentando su eficacia en esferas que no requieren una normatividad mayor por parte de los Estados, pero que resultan cruciales para las comunidades fronterizas.

\section{El papel de los Comités de Frontera, Integración y Desarrollo Fronterizo en la integración chileno-argentina}

Después de décadas de desencuentros entre Chile y Argentina, que incluso estuvieron a punto de desencadenar un conflicto bélico el año 1978, a partir de la década de 1990 con el proceso de redemocratización, ambos países reimpulsaron sus relaciones bilaterales, resolviendo casi la totalidad de sus disputas territoriales bajo los gobiernos de los

2 Comité Pedro Juan Caballero (Paraguay)-Ponta Porá (Brasil). Comité Salto del Guirá (Brasil) -Guiará (Paraguay). Comité Ciudad del Este (Paraguay) -Foz de Iguazú (Brasil). Desde 1979 bajo denominación de Comité Permanente de Frontera Ciudad del Este - Foz de Iguazú con objetivos referidos al tráfico internacional. Comité Posadas (Argentina) -Encarnación (Paraguay) febrero 1985. Comité Clorinda (Argentina) -Colonia Falcón (Paraguay) marzo 1985. Comité Formosa (Argentina) -Alberdi (Paraguay) junio 1985, al que se ha asociado una segunda ciudad Argentina: Colonia Cano. Comité Bermejo (Argentina) -Pilar (Paraguay) julio 1986. Comité de Frontera para el Sistema del Cristo RedentorCaracoles, abril 1987, opera en las circunscripciones consulares de Mendoza - Valparaíso. Comité Río Gallego-Punta Arenas (1989). Comité Bariloche-Puerto Montt. Comité Salta-Antofagasta. Comité Gualeguaychú (Argentina) - Fray Bentos (Uruguay). Comité Concordia (Argentina) -Salto (Uruguay). Comité Colón (Argentina) -Paysandú (Uruguay), Comité La Quiaca -Villazón. Comité Yacuiba -Pocitos Comité Paso de los Libres-Uruguayana. Comité Puerto Iguazú-Foz do Iguazú. 
presidentes Menem en Argentina (1989-1995) y Aylwin en Chile (1990-1994), lo que significó la resolución de 22 litigios. ${ }^{3}$

Durante la última década, las relaciones vecinales han gozado de muy buena salud, incluso en momentos en que hemos asistido a cambios de gobierno y de corrientes ideológicas en ambos países. Es así como durante el primer gobierno del presidente Piñera en Chile (20102014), estas relaciones mantuvieron su matriz original, signada por el retorno a la democracia en Chile y la necesidad de retomar la confianza y fortalecer las relaciones con Argentina.

Durante los gobiernos de los presidentes Néstor Kirchner y Cristina Fernández en Argentina, el espíritu integrador reinaugurado por los ex presidentes Aylwin y Menem en democracia se acentuó, impulsado principalmente por la afinidad ideológica entre los gobiernos de ambos países (Lagos-Kirchner, Bachelet-Fernández), incluso a pesar de los roces ocasionados por la crisis del gas del año 2004.

La agenda desarrollada por los gobiernos de Fernández y Bachelet estuvo marcada por la necesidad de cambiar el enfoque de las relaciones, desde lo predominantemente económico hacia el énfasis político y social de la integración entre ambos países. Reflejo de ello es el Tratado de Maipú del año 2009, que vino a ratificar la disposición integradora, con el objetivo de enfrentar de manera eficaz los desafíos del desarrollo de cada uno de los países y de América Latina en el marco de la globalización, a través del impulso y profundización de las relaciones económicas y comerciales bilaterales, en pos de lograr atender las crecientes demandas que plantea el desarrollo social y cultural de las comunidades de ambos países.

En tal sentido, es importante destacar la institucionalidad de los Comités de Frontera, Integración y Desarrollo Fronterizo (CFIDF), a los que se les dedicó un artículo del tratado. Estos pueden jugar un rol de primer orden al incorporar el paradigma de la descentralización y al mismo tiempo vincular las actividades internacionales a los gobiernos locales y la comunidad organizada.

No obstante la voluntad de ambos países, la agenda actual, dominada por el factor comercial, abre la posibilidad a los CFIDF y a los múltiples actores de la sociedad civil para avanzar en diferentes campos de integración. Es necesario sacar provecho a una relación que ha superado prácticamente todas sus cuestiones fronterizas y cuyas relaciones se han visto intensificadas a pesar de los diferentes gobiernos, lo que habla de una mayor madurez de ambos Estados en materia de institucionalidad bilateral.

La primera expresión de voluntad respecto de la profundización de la relación bilateral en ámbitos específicos a través de comités de frontera se produjo en 1984, con la firma del

3 Queda pendiente el litigio de Campos de Hielo Sur, cuyas negociaciones se han estancado, producto de dificultades metodológicas y del interés por parte de ambos países de avanzar en otras materias y así evitar controversias. 
Tratado de Paz y Amistad. Este fue pensado como un foro en el que los representantes de los servicios aduaneros, migratorios y de vialidad se reunieran con el objeto de coordinar y proponer procedimientos y soluciones ágiles a temas fronterizos.

En el propósito de abarcar temas específicos, se creó en abril de 1987 el primer comité de frontera para el sistema Cristo Redentor-Caracoles mediante el acta de la Segunda Reunión de la Comisión Binacional Argentino-Chilena de Cooperación Económica e Integración Física. Su objetivo fue promover una mayor facilitación y coordinación de medidas operativas concretas que agilizasen y promovieran el tránsito de personas y el tráfico de mercaderías, vehículos, la comunicación y el intercambio turístico, cultural, deportivo, artístico y científico. Como antes se comentó, esta creación correspondió a la etapa de normalización de la relación entre Argentina y Chile y fue calificada como "centro piloto", ya que su experiencia e implementación permitirían extender la operatoria a otros puntos de frontera (Valenciano, 1989).

Actualmente existen los comités Noa (Norte Grande), Comité Agua Negra (Coquimbo-San Juan), Comité Cristo Redentor (Valparaíso-Mendoza), Comité Pehuenche (Región del Maule-Departamentos de Malargüe, San Rafael y General Alvear de la Provincia de Mendoza), Comité Región de Los Lagos (Región de los Lagos-provincias de Neuquén y Río Negro), Comité Integración Austral (regiones de Aysén del General Carlos Ibáñez del Campo y de Magallanes y Antártica Chile-provincias de Santa Cruz y Tierra del Fuego, Antártida e Islas del Atlántico Sur), Comité Las Leñas (Región de O’Higgins-Provincia de Mendoza), Comité Atacalar (Región de Atacama-provincias de Catamarca, La Rioja, Córdoba, Santiago del Estero, Tucumán, Santa Fe).

Los actores subestatales estaban contemplados en los acuerdos solo como posibles invitados, siempre y cuando el tema a tratar lo hiciese pertinente. Sin embargo, paulatinamente fueron pasando a ser foros en los que convergían autoridades subestatales y distintos sectores locales. Por ello, de una reunión entre pocos técnicos, los comités de frontera pasaron a juntar cientos de personas, representativas de ámbitos públicos y privados locales que sobrepasaron el sentido original de la institución.

Si en principio los comités de frontera no estuvieron concebidos para el involucramiento directo de las regiones chilenas y provincias argentinas, el interés de estas devino en su evolución hacia organismos de participación subestatal, lo cual fue seguido por los Estados con el desarrollo de un marco institucional que ampliaba la labor de los comités.

En Chile, los comités dependen de la Dirección de Fronteras y Límites (DIFROL), y de la Dirección de Límites y Fronteras Argentina. Estas son las únicas instancias en que se reconoce oficialmente la actuación de los entes subestatales, según el criterio burocrático que prevalece al interior de los ministerios.

En 1997 se celebró en Santiago el Acuerdo entre la República de Chile y la República Argentina para establecer un reglamento de comités de frontera, en virtud del cual se 
reconoce el funcionamiento de siete comités de frontera (DIFROL, 2017). En 2006 y a través de cartas reversales, se decidió cambiar el nombre de los comités. Estos dejaron de denominarse de Frontera y pasaron a llamarse de Integración, recogiendo la nueva motivación que los impulsaba. De esta manera, los comités se consolidaban como un foro para discutir y proponer soluciones que iban más allá del tránsito fronterizo y que tenían que ver directamente con la integración entre las dos comunidades a ambos márgenes del límite.

El momento cumbre de los foros fue el Tratado de Maipú de Integración y Cooperación, firmado en 2009, que tuvo un enfoque predominante hacia lo político y social y en el que se dedicó un capítulo completo a la participación subestatal, siendo este el más extenso del acuerdo. Allí se consolidó el cambio de carácter de los comités, los cuales pasaban a ser "foros de encuentro y colaboración entre los sectores público y privado de las provincias argentinas y regiones chilenas para promover la integración en el ámbito subnacional, con el apoyo de los organismos nacionales, provinciales, regionales y municipales" (p. 57), y que inclusive podían proponer directamente iniciativas conjuntas a la Comisión Binacional de Cooperación Económica e Integración Física y a la Comisión Binacional de Comercio, Inversión y Relaciones Económicas.

Adicionalmente, el artículo 20 establece que "los comités de integración podrán constituirse por una o más provincias o regiones" (Tratado de Maipú, Art. 20), con lo que se ampliaría el mecanismo más allá de zonas estrictamente fronterizas, generando una instancia de integración descentralizada que puede desarrollar y apoyar iniciativas tan esperadas como los canales bioceánicos. Con ello, los CFIDF recibieron un enorme impulso, reuniendo actualmente a centenares de personas de ambos países para debatir los más diversos temas relativos a la integración, que van desde la infraestructura caminera hasta cuestiones de género, pasando por comisiones legislativas, judiciales y sanitarias, entre otras muchas.

No obstante el espíritu del tratado, especialmente en lo relacionado con los CFIDF, subsisten dificultades que deben ser abordadas tanto al interior de los países como de forma bilateral, tales como la asimetría entre las regiones de Chile y las provincias argentinas. Como plantea Ross:

Debido a la centralización de Chile, los gobiernos subnacionales de este país se han visto debilitados o disminuidos frente a sus pares argentinos, para emprender de manera conjunta aquello que podría cambiar el patrón de dependencia de las relaciones precedentes. Esta limitación es especialmente crítica, en tanto que las sociedades de fronteras y las relaciones transfronterizas, han sido piezas claves de esta relación, especialmente en momentos difíciles. (2017, p. 13)

Asimismo, en términos de interconexión física, las principales obras, como el proyecto del ferrocarril Trasandino y el túnel de baja altura (Las Leñas) quedaron pendientes, y han sido compensadas con el túnel Agua Negra (entre la Provincia de San Juan y la Región de 
Coquimbo), una obra muy importante, pero que no sustituye ni resuelve a la anterior que conectaría Mendoza con la Quinta Región de Chile.

Esto acredita que las autoridades, incluso las más comprometidas con la integración, no alcanzan a comprender que este tipo de propósito superior no debe ser evaluado con criterios de rentabilidad económica, como si se tratara de un proyecto empresarial cualquiera (Ross, 2017), pues contradice el enfoque señalado más arriba de este tratado y el enfoque de la relación que quisieron imprimir los gobiernos de Bachelet y Fernández.

Lo anterior ratifica el interés secundario por los asuntos de frontera en el nivel estatalcentral e impone el desafío a ambos países de hacer efectivo el contenido del tratado en la materia, de modo tal que se fortalezcan las iniciativas de integración subestatal a través de una institucionalidad de integración que supere la sola voluntad política de los Estados e integre a los actores subestatales y privados con una mirada más amplia y descentralizada.

Todo lo anterior no quiere decir que ahora las regiones deben firmar tratados o modificar unilateralmente la política exterior del país (lo que tampoco es posible, dadas las actuales legislaciones de los países). Pero sí deja en claro que para entender de forma cabal la complejidad de los procesos de integración, la dimensión subestatal no puede ser dejada de lado. Antiguos temores al término paradiplomacia -o a lo que ello implica- no tienen lugar en el moderno estudio de las relaciones internacionales, ámbito en el que se requiere seguir incorporando variables para poder entender las vinculaciones cada vez más complejas entre los países, las cuales hace décadas dejaron de limitarse a los acuerdos firmados entre Estados.

\section{Conclusiones}

La experiencia tanto de los tratados de las altas cumbres, así como de los múltiples mecanismos de integración de larga data -que no han logrado alcanzar sus objetivos-, nos hablan de la necesidad de mirar de una manera distinta las relaciones internacionales y, especialmente, la política de integración en nuestra región.

En el escenario global actual, caracterizado por su complejidad y multidimensionalidad, las áreas fronterizas tienen creciente importancia para crear nuevas oportunidades económicas, comerciales y sociales, siendo los procesos de integración regional, los ámbitos de concurrencia natural para su desarrollo político con sus amplios canales de participación: multinacionales y regionales-locales.

En un contexto de amplia y rápida movilidad, las zonas fronterizas deben ser consideradas como el primer eslabón de un proceso de integración mayor, que supere lo estrictamente binacional. Para ello es necesario repensar la centralidad del Estado y la relación vertical que establece con los territorios a la luz del análisis de las relaciones internacionales. 
Repensar la excesiva centralidad del Estado no significa su sustitución, sino plantear la paradiplomacia entendida como una herramienta de la política estatal que al mismo tiempo sirve para alcanzar mayores niveles de autonomía, y además es promotora y facilitadora de iniciativas propias de la sociedad civil. Vista así, la paradiplomacia insinúa ser un mecanismo complementario que para el contexto en el que se desarrollan las relaciones internacionales en nuestra región, resulta más eficiente, dada su flexibilidad, pues le permite adaptarse a las dificultades de nuestra cambiante geografía política.

De tal modo que las ideas de la paradiplomacia ampliada o el pluralismo diplomático, en que el Estado fija las políticas, pero estas puedan ser negociadas con una amplia participación de la sociedad civil, como nueva forma de entender la diplomacia, nos parece el camino adecuado para un escenario donde el Estado ya no resulta suficiente en términos de planteamiento y gestión de relaciones cada vez de mayor alcance, más diversas y más complejas.

La paradiplomacia de actores subestatales y, como planteamos, de la sociedad civil de regiones alejadas de los centros de decisión, a pesar de ocupar un lugar secundario en el pensamiento y las políticas de integración, ha demostrado su eficacia en los Comités de Frontera, Integración y Desarrollo Fronterizo (CFIDF), al incorporar crecientemente a la sociedad civil, la que es capaz de identificar los problemas y/o las oportunidades de zonas colindantes y desarrollar o plantear soluciones permanentes más allá de las contingencias de los Estados y actuar con mayor grado de flexibilidad que estos.

Los CFIDF juegan en la práctica un rol cada vez más importante como mecanismos articuladores entre las zonas fronterizas y los gobiernos centrales, traspasando información para la toma de decisiones. Pero es necesario, a 30 o más años de funcionamiento de estos mecanismos en el Cono Sur, pasar a una nueva etapa, que implicaría dotarlos de mayores atribuciones legales en la toma de decisiones para que no queden como instancias meramente informativas de los organismos centrales.

En este sentido, los Estados miembros de MERCOSUR podrían acordar otorgarles ciertos grados de relación directa a los Criden las comisiones parlamentarias nacionales y regionales, como en el Parlamento del MERCOSUR, en pos de permitir articular y agilizar la aprobación de las propuestas de los CFIDF y así materializar la relación entre lo local, lo nacional y lo regional. Asimismo, ampliar la participación de actores privados, de manera de que puedan asumir temáticas específicas y ampliar el abanico de posibilidades de asuntos a tratar, fomentando así la participación y el empoderamiento de la sociedad civil en el proceso de integración, lo que implica que los actores estatales cumplen una función articuladora y decisoria, pero con una importante influencia de los actores no estatales en la determinación de las mismas.

Si bien la integración ha sido y es una oportunidad para el desarrollo de los países, cabe mencionar algunos elementos que ayudan a explicar sus problemas: la excesiva centralidad 
del proceso en los Estados, los que por diferentes motivos a lo largo de la historia no han sido capaces de alcanzar las metas propuestas, no obstante algunos avances alcanzados, como la constitución de diferentes mecanismos (ALADI, MERCOSUR, UNASUR, PROSUR). Asimismo, la construcción mercadocéntrica ha dejado en segundo plano el aspecto social-cultural -a pesar de los avances en la década del 2000-, entendido este como el cimiento de una integración más vinculada a la sociedad civil.

Históricamente, los latinoamericanos, desde las diversas disciplinas y en especial en el ámbito de las relaciones internacionales, hemos subestimado los márgenes, los que dada nuestra geografía resultan vitales para la interconexión de los territorios, con los beneficios que ello implica tanto en el nivel local como en el global. La experiencia de los estudios de frontera nos demuestra una realidad dinámica que muchas veces difiere de las relaciones que mantienen los países a nivel estatal, ya que en la frontera se entiende mucho mejor la integración desde la cooperación, precisamente porque se practica a diario.

Las unidades subnacionales mantienen una mayor cercanía en relación con las preferencias expresadas por la ciudadanía y, en este sentido, las ciudades pueden convertirse en un elemento esencial en la aglutinación de los intereses prointegracionistas desde abajo. En tal sentido, el aumento de la participación de los grupos de la sociedad civil les permitiría una mejor articulación con las instancias de integración regional a través de la coordinación estatal, de manera que la ciudadanía pueda alcanzar una participación efectiva en la construcción de la agenda de integración, así como en la implementación de los acuerdos. ${ }^{4}$ Asimismo, la integración regional tiene el desafío de asumir la importancia de las interacciones de frontera, haciéndolas más visibles y estimulando a la vez el reconocimiento de factores idiosincrásicos, rescatando la riqueza de las culturas locales y de la diversidad regional que se expresa con más fuerza en territorios alejados de las capitales.

Lo anterior plantea, por un lado, un desafío a la disciplina de las relaciones internacionales en el sentido de considerar la integración regional como un fenómeno complejo en el que interactúan diferentes niveles y no solo a través de la representación estatal en la diplomacia de cumbres. Por otro lado, estimula a comprender que el proceso de integración se inicia en lo local, con la cooperación creciente entre regiones fronterizas, donde deben ser potenciadas las instancias subestatales y aquellas no estatales, propias de la sociedad civil. Para esta inversión de la mirada las comunidades de frontera y los comités están ofreciendo resultados que deben ser incorporados a la reflexión teórica de las relaciones internacionales en el Cono Sur.

4 En esta idea de articulación entre lo local y lo regional es interesante el trabajo que desarrolla ZICOSUR, proyecto integracionista del centro-oeste de América del Sur, que reúne a provincias, departamentos y regiones de Argentina, Bolivia, Brasil, Chile, Paraguay y Perú, cuya función es coordinar propuestas y canalizarlas a los órganos centrales y áreas específicas, como el Asia-Pacífico. 


\section{Referencias}

Aldecoa, F. y Keating, M. (1999). Paradiplomacy in action. The foreign relations of subnational governments. Londres: Frank Cass.

Amilhat Szary, A. L. (2016). Gentes y agentes, condiciones paradiplomáticas de la creación de una frontera móvil. En S. González, N. Cornago y C. Ovando (Eds.), Relaciones transfronterizas y paradiplomacia en América Latina: aspectos teóricos y estudios de casos (pp. 47-72). Santiago: Editorial RIL.

Balassa, B. (1980). Teoría de la integración económica. México, D.F.: UTEHA.

Cornago, N. (2014). Diplomacy decentralized: subnational couples in Latin America. En B. Vassort-Rousset (Ed.), Building sustainable couples in international relations: a strategy towards peaceful cooperation (pp. 20-22). Basingtoke: Palgrave.

Decreto 68/2017. Boletín Oficial de la República Argentina (25 de enero de 2017). Disponible en: https://www.boletinoficial.gob.ar/\#!DetalleNorma/158309/20170126

Dirección de Fronteras y Límites (DIFROL) (2017). Comité de integración ChileArgentina. Recuperado de: http://www.difrol.gob.cl/argentina/comite-deintegracion-chile-argentina.html

Deutsch, K. (1978). The analysis of international relations. Nueva York: Prentice-Hall.

Duchacek, I. (1984). The international dimensions of subnational self government. Publius, 14(4), 5-37. doi: 10.2307/3330188

Duchacek, I. (1990). Perforated sovereignties: towards a typology of new actors in international relations. In H.J. Michelmann y P. Soldatos (Eds.), Federalism and international relations: the role of subnational units (pp. 1-32). Nueva York: Oxford University Press.

Fuentes, C. (2015). La política de coordinación regional. Paradiplomacia en los gobiernos de la Concertación, descentralización y política exterior. En M. Artaza y C. Ross (Eds.), La política exterior de Chile. Del aislamiento a la integración global (pp.281282). Santiago: RIL Editores.

Griffiths, M., O’Callahan, T y Roach, S. (2002). International relations. The key concepts. Nueva York: Routledge.

Hocking, B. (1993). Localizing foreign policy, non-central governments and multilayered diplomacy. Lenders: St. Martin's Press.

Lawrence, R.Z. (1996). Regionalism, multilateralism and deeper integration. Nueva York: The Brookings Institution.

Maira, L. (2010). La política internacional subnacional en América Latina. Buenos Aires: Del Zorzal. 
Meirelles Ribeiro, M. (2008). Ação internacional das entidades subnacionais: teorias e visões sobre a para diplomacia. Revista Cena Internacional, 10(2), 160-184. doi: 132.248.9.34

Morgenthau, H. (1986). Política entre las naciones: la lucha por el poder y la paz. Buenos Aires: G.E.L.

Nye, J. y Keohane, R. (1988). Power and interdependence: world politics in transition. Boston: Little, Brown and Company.

Puig, J.C. (1986). Integración y autonomía de América Latina en las postrimerías del siglo XX. Integración Latinoamericana, (109), 40-62.

Rosenau, J. N. (1997). Demasiadas cosas a la vez. La teoría de la complejidad y los asuntos mundiales. Revista Nueva Sociedad, 148, 70-83.

Ross, C. (2017). ¿En vez de la integración, la alianza estratégica? Working Paper. Instituto de Estudios Avanzados, USACH.

Strange, S. (1996). The retreat of the state: the diffusion of power in the world economy. Cambridge: Cambridge University Press.

Tapia, M. y González, A. (2014). Regiones fronterizas. Migración y los desafíos para los Estados nacionales latinoamericanos. Santiago: Ril Editores-Universidad Arturo Prat.

Tratado de Maipú de Cooperación e Integración Chile-Argentina y sus Protocolos Complementarios. Diario Oficial de la República de Chile, Santiago, Chile, 29 de octubre de 2009. Recuperado de:

https://www.leychile.cl/Navegar?idNorma=1011410\&buscar=tratado+de+maipu

Valenciano, E. (1989). Los Comités de Frontera. Funcionamiento y experiencia. Informe de asesoría. BID-INTAL. Ministerio de Relaciones Exteriores de Chile

Valenciano, E. (1990). Los Comités de Frontera: funcionamiento y experiencia. En: Revista Integración Latinoamericana INTAL, $\mathrm{N}^{\circ} 15$.

Waltz, K. (2008). Realism and international politics. Londres: Routledge. 